\title{
Local dynamics of planar maps with a non-isolated fixed point exhibiting 1-1 resonance
}

\author{
William T. Jamieson ${ }^{1 *}$ and Orlando Merino ${ }^{2}$
}

\section{"Correspondence:}

w.jamieson@snhu.edu

'Department of Mathematics,

S. New Hampshire University,

Manchester, USA

Full list of author information is

available at the end of the article
The Author(s) 2018. This article is distributed under the terms of the Creative Commons Attribution 4.0 International License (http://creativecommons.org/licenses/by/4.0/), which permits unrestricted use, distribution, and reproduction in any medium, provided you give appropriate credit to the original author(s) and the source, provide a link to the Creative Commons license, and indicate if changes were made.

\section{Introduction}

Since the early 1990s, there has been a large amount of activity in the study of autonomous difference equations and discrete dynamical systems, especially in rational difference equations and monotone difference equations. A considerable amount of work has been done in these areas by many authors; see Smith [1], Elaydi [2], Agarwal [3], Ladas [4], and the references therein.

The dynamics of a smooth planar map on a region near a non-hyperbolic fixed point are understood with some notable exceptions. If the characteristic values $\lambda_{1}, \lambda_{2}$ associated with such a fixed point are complex conjugate of each other, then techniques from Kolmogorov-Arnold-Moser (KAM) theory can be used to establish the local dynamical behavior with some exceptions; see [5] for an overview or [6] for an application of the theory. The cases where KAM theory cannot be applied are called strong resonances (see [7], p. 396). A strong resonance occurs when the characteristic values belong to the $\ell$ th roots of unity, for $\ell=1,2,3,4$. A fixed point of a planar map is said to be $1-1$ resonant if the Jacobian matrix of the map at the fixed point is similar to $\left(\begin{array}{ll}1 & 1 \\ 0 & 1\end{array}\right)$.

A fixed point of a planar map is called isolated if there exists a neighborhood of the fixed point that does not contain any other fixed points. In all other cases each fixed point is called non-isolated. If a smooth planar map has a one-dimensional curve consisting entirely of fixed points, then at least one of the associated characteristic values at the fixed points must be equal to 1 . This can be seen by translating the fixed point to the origin, and then performing a local change of coordinates to map the curve of fixed points to one of the axes. Then a standard basis vector is an eigenvector of the Jacobian matrix of the conjugate map at the fixed point, with associated eigenvalue 1.

\begin{abstract} a neighborhood of non-isolated 1-1 resonant fixed points for planar maps that are
\end{abstract}

MSC: $39 A 05 \cdot 39 A 30$

Keywords: Planar differenc
fixed point; Local behavior 
In a 1992 paper [8], Casasayas, Fontich, and Nunes studied the center manifolds of planar $C^{r}$ maps, including real analytic maps, with non-isolated 1-1 resonant fixed points. In [8], the authors proved the existence of invariant curves through such a fixed point under the assumption that the map is in a normal form. However, the complete dynamical behavior near non-isolated 1-1 resonant fixed points was not addressed.

The main purpose of this paper is to give a complete classification of all possible dynamical behavior scenarios valid in a neighborhood of non-isolated 1-1 resonant fixed points for planar maps that are real analytic. Before presenting preliminary material, below we provide examples of maps with a non-isolated 1-1 resonant fixed point:

Example 1 Clark-Kulenović [9] and Clark-Kulenović-Selgrade [10] studied a class of maps that includes the following:

$$
\begin{aligned}
& x_{n+1}=\frac{x_{n}}{a+y_{n}}, \\
& y_{n+1}=\frac{y_{n}}{1+x_{n}},
\end{aligned}
$$

In a population dynamics modeling setting, $x_{n}$ and $y_{n}$ may represent the population levels of two competing species, and $x_{n+1}$ and $y_{n+1}$ represent the population levels one generation later. Map (1) has a continuum of equilibrium points. In fact, every point $(0, y)$ is a fixed point of system (1), and only those points are fixed points [9]. The characteristic values at a point $(0, y)$ are $1 /(a+y)$ and 1 . Thus all fixed points are non-hyperbolic with one characteristic value $\lambda<1$ if $y>1-a$, and non-hyperbolic with one characteristic value $\lambda>1$ if $y<1-a$. Clark and Kulenović did not discuss dynamical behavior near the $1-1$ resonant fixed point; see Fig. 1 .

Non-isolated 1-1 resonant fixed points occur as bifurcation points of host-parasitoid models in mathematical biology. A general host-parasitoid model has the form [11]

$$
\begin{aligned}
& x_{n+1}=c y_{n}\left(1-g\left(x_{n}, y_{n}\right)\right), \\
& y_{n+1}=\lambda y_{n} g\left(x_{n}, y_{n}\right),
\end{aligned} \quad n=0,1, \ldots, x_{0}, y_{0} \in[0, \infty),
$$

where $x_{n}$ and $y_{n}$ represent the populations of the parasitoid and the host at time $n$, respectively. The parameters $\lambda$ and $c$ represent the intrinsic growth rate of the host and the number of viable eggs laid by a single parasitoid. The function $g:[0, \infty)^{2} \mapsto[0,1]$ represents the probability that a host escapes being infested by a parasitoid. If system (2) is to

Figure 1 Clark-Kulenović system (1) with $a=0.5$ simulations suggest the existence of an invariant curve (dashed) that passes through $(0,0.5)$, which appears to be a boundary of regions with different dynamical behavior. The point in the center of the plot is $(0,0.5)$ and the solid vertical line consists entirely of fixed points. The curves depict paths followed by orbits under iteration of the map of system (1)

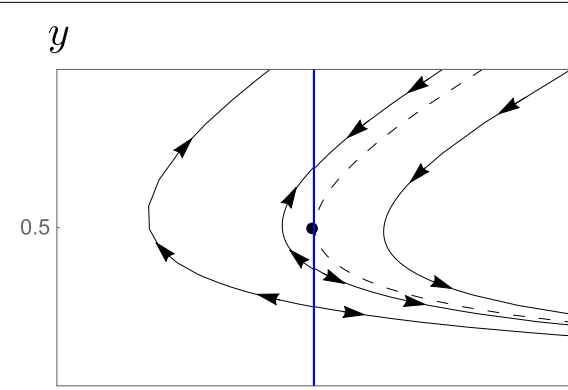


be a reasonable model, we should choose $g(0, y)=1$ for all $y$, since all hosts will escape infestation if the parasitoid population is zero. Then if the intrinsic growth rate of the host, $\lambda$, is equal to 1 , the equilibrium points of system (2) are of the form $(0, y)$ for all $y \in[0, \infty)$. Further, $g(0, y)=1$ for all $y$ implies that $D_{y} g(0, y)=0$ for all $y$. Then the Jacobian matrix of the associated map at $(0, y)$ is

$$
\left(\begin{array}{cc}
-c y D_{x} g(0, y) & -c y D_{y} g(0, y) \\
y D_{x} g(0, y) & 1+y D_{y} g(0, y)
\end{array}\right)=\left(\begin{array}{cc}
-c y D_{x} g(0, y) & 0 \\
y D_{x} g(0, y) & 1
\end{array}\right) .
$$

If there exists a $\bar{y} \in[0, \infty)$ such that $\bar{y} D_{x} g(0, \bar{y})=-\frac{1}{c}$, then $(0, \bar{y})$ is a non-isolated $1-1$ resonant fixed point. The existence of a solution to the equation $\bar{y} D_{x} g(0, \bar{y})=-\frac{1}{c}$ is satisfied by the classical host-parasitoid models, including Nicholson-Bailey where $g(x, y)=e^{-a x}$, Rogers where $g(x, y)=e^{\frac{-a x F}{F+a y}}$, and May's host-parasitoid where $g(x, y)=\left(1+\frac{a x}{k}\right)^{-k}$; see [12, $13]$ and [14].

Example 2 D. McArdle and O. Merino [15] considered the Leslie host-parasite model (see also $[16,17])$

$$
\begin{aligned}
& x_{n+1}=\frac{\alpha x_{n}}{1+\beta y_{n}}, \\
& y_{n+1}=\frac{\gamma x_{n} y_{n}}{x_{n}+\delta y_{n}},
\end{aligned}
$$

where $\alpha, \beta, \gamma, \delta \in \mathbb{R}^{+}$. If $\alpha=\beta=\gamma=\delta=1$ in system (4) gives the system

$$
\begin{aligned}
& x_{n+1}=\frac{x_{n}}{1+y_{n}}, \\
& y_{n+1}=\frac{x_{n} y_{n}}{x_{n}+y_{n}}, \quad n=0,1, \ldots, x_{0}, y_{0} \in[0, \infty), x_{0}+y_{0} \neq 0 .
\end{aligned}
$$

It can easily be verified that every point on the positive $(\bar{x}, 0)$ with $\bar{x}>0$ is an equilibrium point of system (5), and that the Jacobian matrix at $(\bar{x}, 0)$ has the form $\left(\begin{array}{cc}1 & -\bar{x} \\ 0 & 1\end{array}\right)$, so the positive $x$ semi-axis consists entirely of non-isolated $1-1$ resonant equilibrium points.

We now give an overview of the manuscript. Section 2 reviews known results for invariant manifolds of non-isolated 1-1 resonant fixed points. In Sect. 3 the set of fixed points near a non-isolated 1-1 resonant fixed point is shown to be a curve in Theorem 1 . Also, we introduce normal forms, and conditions are given in Theorem 2 for the normal form to exist. Theorem 2 also gives information relevant to the normal form for application of our dynamical behavior results without the need to have an explicit normal form, which is useful because finding a normal form may be difficult in practice. Further, Sect. 3 introduces sectors, which we will use to describe dynamical behavior in the statement of the main result of this paper, Theorem 3, which is stated in Sect. 4 together with further discussion of examples and applications. The proof of Theorem 3, given in Sect. 5.4, relies on Propositions 1 and 2, whose proofs are presented in Sects 5.2 and 5.3.

\section{Invariant manifolds}

The following result, which is a combination of Theorems 2.1 and 3.1 in [8], characterizes invariant curves through a non-isolated 1-1 resonant fixed point in the real analytic case. 


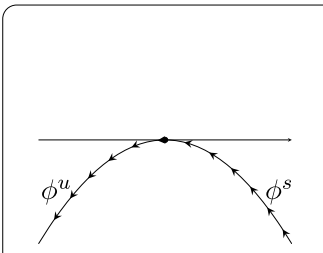

(a)

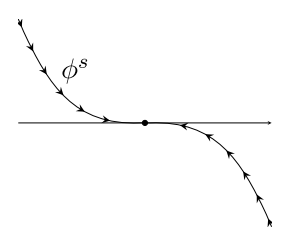

(b)
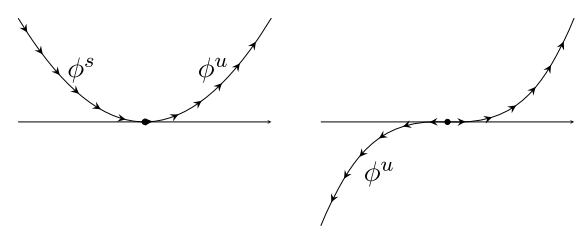

(c)

(d)

Figure 2 The invariant curves guaranteed by Theorem A. The horizontal line consists entirely of fixed points in a neighborhood $U$ of the origin (the black dot)

This characterization is key for our main result, Theorem 3. From now on, the partial differentiation operators are denoted $D_{x}$ and $D_{y}$, the Jacobian matrix of a map $F$ at $(x, y)$ is denoted $D F(x, y)$, and $F_{\ell}$ is the $\ell$ th coordinate function of $F$ for $\ell=1,2$.

Theorem A (Casasayas et al.) Let $U$ be an open set of $\mathbb{R}^{2}$ containing the origin and let $F: U \rightarrow \mathbb{R}^{2}$ be a real analytic map such that (i) $F(x, 0)=(x, 0)$ for $(x, 0) \in U$, (ii) $D F(0,0)=\left(\begin{array}{ll}1 & 1 \\ 0 & 1\end{array}\right)$, and (iii) there exists $n \geq 2$ such that $D_{x}^{i} D_{y}^{j} F(0,0)=0$ for $1<i+j<n$, and $D_{x}^{n-1} D_{y} F_{2}(0,0) \neq 0$. Let $b=\frac{1}{(n-1) !} D_{x}^{n-1} D_{y} F_{2}(0,0)$. Then local stable and unstable manifolds of the origin exist according to cases (a)-(d) given below. When they exist, they are the graph of functions that are real analytic on any subinterval of their domain that excludes 0 .

(a) If $b<0$ and $n$ is even, then there exist $\delta>0, \phi^{s}:[0, \delta) \rightarrow(-\infty, 0]$, and $\phi^{u}:(-\delta, 0] \rightarrow(-\infty, 0]$, respectively, such that $W_{\mathrm{loc}}^{s}(\delta)=\left\{\left(x, \phi^{s}(x)\right): x \in[0, \delta)\right\}$ and $W_{\mathrm{loc}}^{u}(\delta)=\left\{\left(x, \phi^{u}(x)\right): x \in(-\delta, 0]\right\}$.

(b) If $b<0$ and $n$ is odd, then there exist $\delta>0$ and $\phi^{s}:(-\delta, \delta) \rightarrow \mathbb{R}$, such that $W_{\text {loc }}^{s}(\delta)=\left\{\left(x, \phi^{s}(x)\right): x \in(-\delta, \delta)\right\}$.

(c) If $b>0$ and $n$ is even, then there exist $\delta>0, \phi^{s}:(-\delta, 0] \rightarrow[0, \infty)$, and $\phi^{u}:[0, \delta) \rightarrow[0, \infty)$, respectively, such that $W_{\mathrm{loc}}^{s}(\delta)=\left\{\left(x, \phi^{s}(x)\right): x \in(-\delta, 0]\right\}$ and $W_{\mathrm{loc}}^{u}(\delta)=\left\{\left(x, \phi^{u}(x)\right): x \in[0, \delta)\right\}$.

(d) If $b>0$ and $n$ is odd, then there exist $\delta>0$ and $\phi^{u}:(-\delta, \delta) \rightarrow \mathbb{R}$, such that $W_{\text {loc }}^{u}(\delta)=\left\{\left(x, \phi^{u}(x)\right): x \in(-\delta, \delta)\right\}$.

Furthermore, the only invariant curves through $(0,0)$ are given by (a)-(d) and hypothesis (i). In all cases, $\phi^{s, u}(x) \sim b x^{n} / n$ as $x \rightarrow 0$.

Figure 2 depicts the scenarios described in Theorem A. The authors of [8] did not treat the case where hypothesis (iii) of Theorem A is not satisfied. Since such case is a possible dynamical scenario, we supplement Theorem A with the following result, which precludes the existence of invariant curves through the 1-1 resonant point other than the curve of fixed points.

Theorem B Let $U$ be an open set of $\mathbb{R}^{2}$ containing the origin and let $F: U \rightarrow \mathbb{R}^{2}$ be a real analytic map such that (i) $F(x, 0)=(x, 0)$ for $(x, 0) \in U$, and (ii) $D F(0,0)=\left(\begin{array}{ll}1 & 1 \\ 0 & 1\end{array}\right)$. If $D_{x}^{n-1} D_{y} F_{2}(0,0)=0$ for all $n \geq 2$, then there exists one and only one invariant curve through the origin. Such curve consists of fixed points of $F$.

The proof of Theorem B is presented as Case 5 of Theorem 3 in Sect. 5.4.

The invariant curves in Theorems $\mathrm{A}$ and $\mathrm{B}$ along with the $x$-axis, split a neighborhood of $(0,0)$ into either two or four regions, which we will term sectors. In Definition 3 , we in- 
troduce four types of sectors, namely hyperbolic, attracting parabolic, repelling parabolic, and elliptic. Our main theorem, Theorem 3, will classify the dynamical behavior near nonisolated 1-1 resonant fixed points in terms of these sectors.

\section{Curves of fixed points, normal forms, and sectors}

Suppose $S$ is a real analytic map defined on a neighborhood of a 1-1 resonant fixed point. In this section we shall assume, without loss of generality, that the fixed point is $(0,0)$, $D S(0,0)=\left(\begin{array}{ll}1 & 1 \\ 0 & 1\end{array}\right)$, and there exist a neighborhood $U$ of $(0,0)$ and real analytic functions $f(x, y)$ and $g(x, y)$ on $U$ such that

$$
S(x, y)=(x+y+f(x, y), y+g(x, y)), \quad(x, y) \in U,
$$

and

$$
f(0,0)=g(0,0)=D_{x} f(0,0)=D_{y} f(0,0)=D_{x} g(0,0)=D_{y} g(0,0)=0 .
$$

The next result states that near any 1-1 resonant non-isolated fixed point, the set of fixed points of a real analytic planar map is a real analytic curve.

Theorem 1 Let $W$ be an open set of $\mathbb{R}^{2}$ containing the origin and let $S: W \rightarrow \mathbb{R}^{2}$ be a real analytic map for which $(0,0)$ is a non-isolated fixed point, and such that $D S(0,0)=\left(\begin{array}{ll}1 & 1 \\ 0 & 1\end{array}\right)$. Then there exist a neighborhood $U \subset W$ of the origin such that the set $\mathcal{F}$ of fixed points of $S$ in $U$ is a real analytic curve through $(0,0)$. The curve $\mathcal{F}$ is tangential at $(0,0)$ to the one-dimensional eigenspace of $D S(0,0)$.

Proof Let $f$ and $g$ be real analytic functions as in (6) and (7). In particular, $1+D_{y} f(x, y) \neq 0$ for $(x, y)$ close enough to $(0,0)$. Set $F(x, y)=y+f(x, y)$. If $(x, y)$ is a fixed point of $S$, then necessarily $F(x, y)=0$. Then by these considerations and the real analytic implicit function theorem (Theorem 1.8.3 in [18]), there exists a neighborhood $I \subset \mathbb{R}$ of 0 and a real analytic function $y=\varphi(x)$ on $I$ such that, for $x \in I, 1+D_{x} f(x, \varphi(x)) \neq 0, F(x, \varphi(x))=0$, and

$$
\varphi^{\prime}(x)=\frac{-D_{x} f(x, \varphi(x))}{1+D_{y} f(x, \varphi(x))}, \quad x \in I
$$

Thus there exists a neighborhood $U$ of the origin, $U \subset W$, such that every fixed point of $S$ in $U$ must be of the form $(x, \varphi(x))$ for some $x$ near 0 . We now show that points of the form $(x, \varphi(x))$ satisfy $g(x, \varphi(x))=0$, and consequently they are fixed points of $S$. Since $(0,0)$ is a non-isolated fixed point, there exists a sequence $\left(x_{n}, y_{n}\right)$ of fixed points that converges to $(0,0)$. By the previous discussion, $y_{n}=\varphi\left(x_{n}\right)$ for all $n$ larger than some $N \in \mathbb{N}$, and thus we have $g\left(x_{n}, \varphi\left(x_{n}\right)\right)=0$, for all $n \geq N$. Then $g(\cdot, \varphi(\cdot))$ is a real analytic function on $I$ that has a sequence of fixed points that accumulates in the interior of $I$. By Corollary 1.2.6 in [18], $g(x, \varphi(x))=0$ for every $x \in I$. Finally, relations (7) and $(8)$ give $\varphi^{\prime}(0)=\frac{-D_{x} f(0,0)}{1+D_{y} f(0,0)}=0$, which is the last statement in the theorem.

The definitions below are an adaptation of the normal form for continuous dynamical systems used by Gubar in [19]. 
Definition 1 A real analytic map $T$ defined on a neighborhood $V \subset \mathbb{R}^{2}$ of the origin is said to be in normal form if there exist real analytic functions $Q$ and $R$ where either $Q(\cdot)$ is the zero function, or $Q(0) \neq 0$ and there exists an integer $\ell \geq 1$ such that

$$
T(x, y)=\left(x+y, y+Q(x) x^{\ell} y+R(x, y) y^{2}\right) \quad \text { for }(x, y) \in V .
$$

Note that, as a consequence of Eq. (9), any fixed point $(\bar{x}, \bar{y})$ of $T(x, y)$ in $V$ must have $\bar{y}=0$.

Definition 2 Let $W$ be an open set in $\mathbb{R}^{2}$ containing a point $(\bar{x}, \bar{y})$, and $S: W \rightarrow \mathbb{R}^{2}$ be a real analytic map for which $(\bar{x}, \bar{y})$ is a non-isolated, $1-1$ resonant fixed point. A planar map $T$ is a normal form of $S$ relative to $(\bar{x}, \bar{y})$ and $W$ if $T$ is in normal form and there exists a real analytic change of coordinates under which $(\bar{x}, \bar{y})$ is mapped to the origin and the map $S$ conjugates to $T$ on $W$.

Remark 1 Normal forms are preserved under certain conjugations. Suppose a map $T$ is as in (9). Conjugation of $T$ by $\Theta_{a}(x, y):=(a x, a y)$ (where $a \in \mathbb{R} \backslash\{0\}$ ) gives the map

$$
T_{a}(x, y)=\left(x+y, y+a^{\ell} Q(a x) x^{\ell} y+a R(a x, a y) y^{2}\right), \quad \text { for }(x, y) \in \frac{1}{a} V
$$

Clearly $T_{a}$ is in a normal form.

Remark 2 A map $T$ in normal form satisfies the hypotheses of either one of Theorems A and B. Indeed, (i) and (ii) are clearly true, and if (iii) of Theorem A does not hold, then Theorem B applies.

The following result shows that every real analytic map $S: W \rightarrow \mathbb{R}^{2}$ for which $(\bar{x}, \bar{y})$ is a non-isolated, type 1-1 resonant fixed point has a normal form (9). Further, the result shows that the term $Q(x) x^{\ell} y$ of a normal form (9) can be found without the need to calculate the normal form if the curve of fixed points has a known real analytic formula. This will be useful in applications for verification of the hypotheses of Theorem 3 in Sect. 4.1.

Theorem 2 Let $W$ be an open set of $\mathbb{R}^{2}$ containing the origin and let $S: W \rightarrow \mathbb{R}^{2}$ be a real analytic map for which $(0,0)$ is a non-isolated fixed point, and such that $D S(0,0)=\left(\begin{array}{ll}1 & 1 \\ 0 & 1\end{array}\right)$. Then there exist a neighborhood $U \subset W$ of the origin and a normal form of $S$ relative to $(0,0)$ and $U$,

$$
T(x, y)=\left(x+y, y+Q(x) x^{\ell} y+R(x, y) y^{2}\right) \quad \text { for }(x, y) \in V
$$

where $V$ is a neighborhood of the origin.

Suppose the set $\mathcal{F}$ offixed points of $S$ in $U$ are of the form $\{(x, \xi(x)): x \in I\}$, where $I$ is an interval containing 0 and $\xi$ is a real analytic function on I satisfying $\xi(0)=0$ and $\xi^{\prime}(0)=0$.

Then the real analytic functions $f$ and $g$ from (6) and (7) satisfy

$$
Q(x) x^{\ell}=\left.D_{y}(g(x, y)-\xi(x+y+f(x, y)))\right|_{y=\xi(x)}, \quad x \in I .
$$


In particular, if $\xi(x)=0$ for $x \in I$, then

$$
Q(x) x^{\ell}=D_{y} g(x, 0), \quad x \in I .
$$

Remark 3 In Theorem 3, the local dynamical behavior of (6) will be classified by the sign of $Q(0)$ and parity of $\ell$, which can be found by taking a power series expansion of (12) about $x=0$. For each $n \in \mathbb{N}$, let $\xi_{n}(x)$ be the power series of $\xi(x)$ truncated to include terms up to the $n$th power. In general, the sign of $Q(0)$ and parity of $\ell$ in the lowest-order term of the power series about $x=0$ of using $y=\xi(x)$ in Eq. (12) are not the same as those found using $y=\xi_{n}(x)$, but are the same if $n$ is chosen large enough. In practice, one should choose $n$ such that the sign of $Q(0)$ and parity of $\ell$ using $y=\xi_{n}(x)$ in (12) do not change when compared to those found using $y=\xi_{n+1}(x)$.

Proof The map $\Theta(x, y):=(x, y+\xi(x))$, defined on a neighborhood $U$ of the origin chosen so that $\Theta(U) \subset W$, is real analytic, $1-1$, and satisfies $\Theta(0,0)=(0,0)$ and $\Theta^{-1}(x, y)=(x, y-\xi(x))$ for $(x, y)$ near the origin. The conjugate map of $S$ through $\Theta$ is given by

$$
F(x, y)=\Theta^{-1} S \Theta=\left(\begin{array}{c}
x+y+\xi(x)+f(x, y+\xi(x)) \\
y+\xi(x)+g(x, y+\xi(x))-\xi(x+y+\xi(x)+f(x, y+\xi(x)))
\end{array}\right) .
$$

The map $F(x, y)$ satisfies

$$
F(x, 0)=(x, 0), \quad(x, 0) \in U
$$

By the real analytic character of $F(x, y)$ and by (15), there exist real analytic functions $v$ and $\psi$ such that

$$
\left\{\begin{array}{l}
\tilde{x}=x+y+y v(x, y), \\
\tilde{y}=y+y \psi(x, y)
\end{array} \quad(x, y) \in U .\right.
$$

Set

$$
z=y+v(x, y) y \quad \text { and } \quad \tilde{z}=\tilde{y}+v(x, \tilde{y}) \tilde{y} .
$$

Combine (16) and (17) to obtain

$$
\left\{\begin{array}{l}
\tilde{x}=x+z, \\
\tilde{z}=(y+y \psi(x, y)) v(x, y+y \psi(x, y))+y+y \psi(x, y),
\end{array} \quad(x, y) \in U .\right.
$$

The function $v$ satisfies $v(0,0)=0$, so there exists a neighborhood $U^{\prime} \subset U$ of the origin such that

$$
v(x, 0) \neq-1 \quad \text { for }(x, 0) \in U^{\prime} .
$$

The latter relation, the first equation of (17), and the real analytic implicit function theorem (Theorem 1.8.3 in [18]) imply that there exist a neighborhood $V \subset U^{\prime}$ of the origin 
and a real analytic function $h$ on $V$ such that $y=h(x, z)$ for $(x, z) \in V$. From substituting $y=h(x, z)$ in (18), the latter may be rewritten as

$$
\left\{\begin{array}{l}
\tilde{x}=x+z, \\
\tilde{z}=\tilde{h}(x, z)
\end{array} \quad(x, y) \in V,\right.
$$

where $\tilde{h}(x, z)$ is a real analytic function on $V$. Now $\tilde{h}(x, 0)=0$ for $(x, 0) \in V$, by the choice of $U^{\prime} \supset V$ and by the first equation of (17). Thus there exists $\hat{h}$ real analytic on $V$ such that

$$
\tilde{h}(x, z)=z \hat{h}(x, z) \quad \text { for }(x, z) \in V \text {. }
$$

Next we verify that $\hat{h}(0,0)=1$. By (17) and (19), $z=0$ if and only if $y=0$, so (17), (20) and (21) imply that, for $(x, z) \in V$ with $z \neq 0$,

$$
\hat{h}(x, z)=\frac{\tilde{z}}{z}=\frac{\tilde{y}(1+v(\tilde{x}, \tilde{y}))}{y(1+v(x, y))}=\frac{(1+\psi(x, y))(1+v(\tilde{x}, \tilde{y}))}{(1+v(x, y))} .
$$

Since the last term in (22) is a continuous function of $(x, z)$ near the origin and it has the value 1 there, it follows that $\hat{h}(0,0)=1$. Now, $\hat{h}(x, 0)-1$ is a real analytic function of $x$ near zero. Write $\hat{h}(x, 0)-1=Q(x) x^{\ell}$, where the real analytic function $Q$ is either the zero function, or $\ell \geq 1$ and $Q(0) \neq 0$. Then $\hat{h}(x, z)-1-Q(x) x^{\ell}$ is real analytic and we may write $\hat{h}(x, z)=1+Q(x) x^{\ell}+R(x, z) z$, where $R$ is real analytic. Then equations (20) and (21) give a normal form (9), that is, that Eq. (14) can be written as

$$
\left\{\begin{array}{l}
\tilde{x}=x+z, \\
\tilde{z}=z+Q(x) x^{\ell} z+R(x, z) z^{2},
\end{array} \quad(x, z) \in V,\right.
$$

by setting $z=y+\varphi(x)+f(x, y+\varphi(x))=y+y v(x, y)$ and $\tilde{z}=\tilde{y}+\tilde{y} v(\tilde{x}, \tilde{y})$. These relations, (16), and (23) give, for $(x, 0) \in V$,

$$
\begin{aligned}
Q(x) x^{\ell} & =\lim _{z \rightarrow 0} \frac{\tilde{z}-z}{z} \\
& =\lim _{y \rightarrow 0} \frac{\tilde{y}+v(x, \tilde{y}) \tilde{y}-y-v(x, y) y}{y+v(x, y) y} \\
& =\lim _{y \rightarrow 0} \frac{y+y \psi(x, y)+v(x, y+y \psi(x, y))(y+y \psi(x, y))-y-v(x, y) y}{y+v(x, y) y} \\
& =\lim _{y \rightarrow 0} \frac{\psi(x, y)+v(x, y+y \psi(x, y))(1+\psi(x, y))-v(x, y)}{1+v(x, y)} \\
& =\frac{\psi(x, 0)+v(x, 0)(1+\psi(x, 0))-v(x, 0)}{1+v(x, 0)} \\
& =\psi(x, 0) \\
& =\left.D_{y}(\xi(x)+g(x, y+\xi(x))-\xi(x+y+\xi(x)+f(x, y+\xi(x))))\right|_{y=0} \\
& =D_{y} g(x, \xi(x))-\xi^{\prime}(x+\xi(x)+f(x, \xi(x))) \cdot\left(1+D_{x} f(x, \xi(x))\right) \\
& =\left.D_{y}(g(x, y)-\xi(x+y+f(x, y)))\right|_{y=\xi(x)} .
\end{aligned}
$$


Differentiating $g(x, y)=y \psi(x, y)$ with respect to $y$ yields $D_{y} g(x, y)=\psi(x, y)+y D_{y} \psi(x, y)$. If $\xi(x)=0$ for all $x \in U$, then $\psi(x, 0)=D_{y} g(x, 0)$, and thus (13) follows.

\subsection{Normal form of the inverse map}

Lemma 1 Let $T$ be a map in normal form with $Q$ and $\ell$ as in (9). If $(-1)^{\ell+1} Q(0)<0$, then $T^{-1}$ has a normal form $\tilde{T}$ such that $\tilde{Q}(0)>0$.

Proof With the involution $\Theta(x, y)=(-x, y)$, the map $T$ conjugates to $(u, v)=\left(\Theta^{-1} T \Theta\right)(x$, $y)=\left(x-y, y+Q(-x)(-x)^{\ell} y+R(-x, y) y^{2}\right)$. By the inverse function theorem, $\Theta^{-1} T \Theta$ has an inverse in a neighborhood of the origin, whose Jacobian matrix at the origin is $\left(\begin{array}{ll}1 & 1 \\ 0 & 1\end{array}\right)$, and which has the form

$$
\left(\Theta T^{-1} \Theta^{-1}\right)(u, v)=(x, y)=(u+v+H(u, v), v+H(u, v))
$$

for some real analytic function $H(u, v)=\Gamma(x, y):=-Q(-x)(-x)^{\ell} y-R(-x, y) y^{2}$. The fixed points of the map $T$ (and thus of $T^{-1}$ ) have the form $(x, 0)$, which were unaffected by reflection over the $y$-axis, so points of the form $(u, 0)$ are fixed points of $\Theta T^{-1} \Theta^{-1}$, and thus $\Phi(u, 0)=0$. By Theorem $2, \Theta T^{-1} \Theta^{-1}$ is conjugate to a map $\tilde{T}$ such that $\tilde{T}$ is in form (9). Taking the partial derivative of both sides of the equation $H(u, v)=\Gamma(u+v+H(u, v), v+$ $H(u, v))$ with respect to $v$, solving for $D_{v} H(u, v)$, and evaluating at the point $(u, 0)$ yields

$$
D_{v} H(u, 0)=\frac{D_{v} \Gamma(u, 0)}{1+D_{v} \Gamma(u, 0)}=-Q(0)(-u)^{\ell}+O(1) u^{\ell+1} .
$$

By (25) we can surmise from Theorem 2 that $T$ and any normal form of $T^{-1}$ have the same value of $\ell$, and that $(-1)^{\ell+1} Q(0)<0$ implies that $\tilde{Q}(0)>0$.

An implication of the proof of Lemma 1 is that the change of coordinates from $T^{-1}$ to the normal form $\tilde{T}$ flips the horizontal axis about the origin, and maps (locally) each vertical semi-axis into itself (see Corollary 1). Let

$$
\begin{array}{ll}
\mathbb{X}_{+}:=\{(x, 0): x \geq 0\}, & \mathbb{X}_{-}:=\{(x, 0): x \leq 0\} \\
\mathbb{Y}_{+}:=\{(0, y): y \geq 0\}, & \mathbb{Y}_{-}:=\{(0, y): y \leq 0\}
\end{array}
$$

For $\delta>0$, denote with $\mathcal{B}_{\delta}$ the set $\{(x, y):-\delta \leq x \leq \delta,-\delta \leq y \leq \delta\}$ and for $\ell=1, \ldots, 4$, denote with $\mathcal{Q}_{\ell}$ the usual closed quadrants with respect to the origin.

Corollary 1 Let $T$ and $\tilde{T}$ be as in Lemma 1 . Then, for each $\delta>0$, a conjugacy map $\Theta$ for which $T^{-1}=\Theta^{-1} \tilde{T} \Theta$ and $a \delta^{\prime}>0$ can be chosen to satisfy any of the following relations:

$$
\begin{array}{ll}
\Theta\left(\mathbb{X}_{+} \cap \mathcal{B}_{\delta^{\prime}}\right) \subset \mathbb{X}_{-} \cap \mathcal{B}_{\delta}, & \Theta\left(\mathbb{X}_{-} \cap \mathcal{B}_{\delta^{\prime}}\right) \subset \mathbb{X}_{+} \cap \mathcal{B}_{\delta} \\
\Theta\left(\mathbb{Y}_{+} \cap \mathcal{B}_{\delta^{\prime}}\right) \subset \mathbb{Y}_{+} \cap \mathcal{B}_{\delta}, & \Theta\left(\mathbb{Y}_{-} \cap \mathcal{B}_{\delta^{\prime}}\right) \subset \mathbb{Y}_{-} \cap \mathcal{B}_{\delta}
\end{array}
$$

Proof The conjugacy map $\Theta$ is the composition of two mappings - the first mapping is a reflection over the $y$-axis. Clearly the upper-and lower-half planes and the $x$-axis are invariant under this reflection. The second mapping is the change of coordinates $w=y+$ 


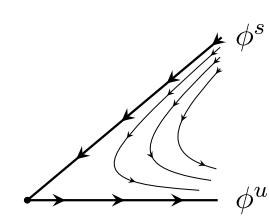

(a)

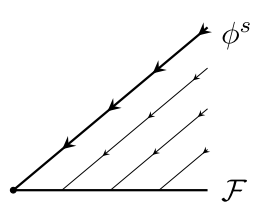

(b)

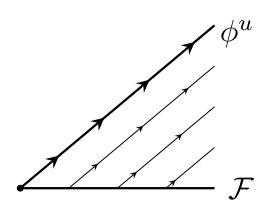

(c)

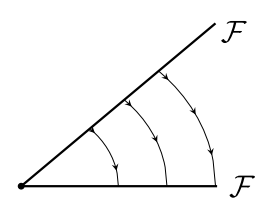

(d)

Figure 3 Illustrations of (a) a hyperbolic sector, (b) an attracting parabolic sector, (c) a repelling parabolic sector, and (d) an elliptic sector. The arrows represent the direction of the trajectories of the orbits. Here $\phi^{s}$ and $\phi^{u}$ are the curves in Theorem $A$, and $\mathcal{F}$ is the curve of fixed points

$H(x, y)$. Since $H(x, 0)=0$ for all $(x, 0) \in V$ and $H$ is real analytic, $H(x, y)=y \tilde{H}(x, y)$ for some real analytic $\tilde{H}$ in $V$, and $V$ can be chosen small enough so that $|H(x, y) / y|<1$. Thus, if $y>0$, then $w=y+H(x, y)=y\left(1+\frac{H(x, y)}{y}\right)>0$. The proof for $y<0$ is similar.

We now introduce the concept of sectors.

Definition 3 Let $S$ be a planar real analytic map defined on an open set $W$ containing a non-isolated 1-1 resonant fixed point $(\bar{x}, \bar{y})$. Let $T$ be a normal form of $S$ relative to $(\bar{x}, \bar{y})$ and $U \subset W$. If $T$ satisfies the hypotheses of Theorem A, set $\tilde{\Phi}$ to be the curve guaranteed in the conclusion of Theorem A, and if the hypotheses of Theorem A are not met, set $\tilde{\Phi}=\emptyset$. Let $V$ be an open connected neighborhood of $(\bar{x}, \bar{y})$ such that the closure of $V$ is a subset of $U$ and $S^{-1}$ exists on the closure of $V$. Denote with $\mathcal{F}$ the curve of fixed points of $S$ in $V$, and let $\Phi$ denote the image of the set $\tilde{\Phi}$ under the change of coordinates that takes $T$ to $S$.

A connected component of $V \backslash(\Phi \cup \mathcal{F})$ is called a sector of $S$ (relative to $(\bar{x}, \bar{y})$ and $V$ ). Let $\mathcal{R}$ be a sector.

(a) $\mathcal{R}$ is a hyperbolic sector of $S$ relative to $(\bar{x}, \bar{y})$ and $V$ if for every $(x, y) \in \mathcal{R}$ there exist $m, n$ in $\mathbb{N}$ such that $S^{\ell}(x, y) \in \mathcal{R}$ for $-m \leq \ell \leq n$, and $S^{\ell}(x, y) \notin V$ for $\ell=-m-1$ and $\ell=n+1$.

(b) $\mathcal{R}$ is an attracting parabolic sector of $S$ relative to $(\bar{x}, \bar{y})$ and $V$ if for every $(x, y) \in \mathcal{R}$, there exists $m \in \mathbb{N}$ such that $S^{\ell}(x, y) \in \mathcal{R}$ for $-m \leq \ell<\infty, S^{-m-1}(x, y) \notin V$, and $\left\{S^{\ell}(x, y)\right\}_{\ell=0}^{\infty}$ converges to a point in $\mathcal{F} \backslash\{(\bar{x}, \bar{y})\}$.

(c) $\mathcal{R}$ is a repelling parabolic sector of $S$ relative to $(\bar{x}, \bar{y})$ and $V$ if for every $(x, y) \in \mathcal{R}$, there exists $n \in \mathbb{N}$ such that $S^{\ell}(x, y) \in \mathcal{R}$ for $-\infty<\ell<n, S^{n+1}(x, y) \notin V$, and $\left\{S^{-\ell}(x, y)\right\}_{\ell=0}^{\infty}$ converges to a point in $\mathcal{F} \backslash\{(\bar{x}, \bar{y})\}$.

(d) $\mathcal{R}$ is an elliptic sector of $S$ relative to $(\bar{x}, \bar{y})$ and $V$ if there exists an open disk $B((\bar{x}, \bar{y}) ; \delta) \subset V$ such that $S^{\ell}(x, y) \in \mathcal{R}$ for every $(x, y) \in \mathcal{R} \cap B((\bar{x}, \bar{y}) ; \delta)$ and every $\ell \in \mathbb{Z}$, and both $\left\{S^{\ell}(x, y)\right\}_{\ell=0}^{\infty}$ and $\left\{S^{-\ell}(x, y)\right\}_{\ell=0}^{\infty}$ converge to points in $\mathcal{F} \backslash\{(\bar{x}, \bar{y})\}$.

See Fig. 3 for an illustration.

\section{Main result}

\subsection{Statement}

The next theorem is the main result of this paper. It gives a classification of possible dynamic scenarios near a 1-1 resonant fixed point in terms of the curve of fixed points and of the normal form. 


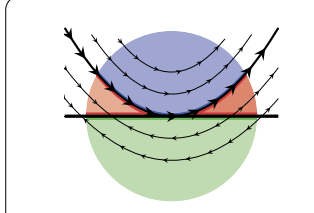

(i) $Q \neq 0, \ell$ is odd: One hyperbolic, two parabolic, and one elliptic sector

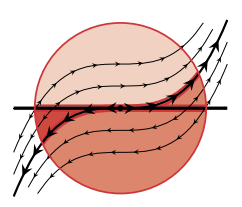

(ii) $Q>0, \ell$ is even: Four parabolic sectors

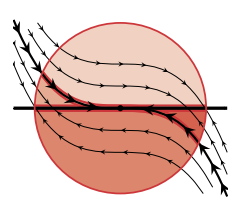

(iii) $Q<0, \ell$ is even:

Four parabolic sectors

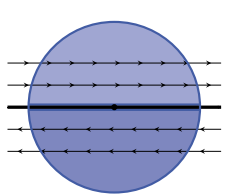

(iv) $Q=0$ : Two hyperbolic sectors

Figure 4 Dynamical behavior near a 1-1 resonant fixed point $(\bar{x}, \bar{y})$, according to Theorem 3; the black dot is $(\bar{x}, \bar{y})$. The thick curves through $(\bar{x}, \bar{y})$ are the invariant curves in Theorems $A$ and $B$. The sectors appear in blue (hyperbolic), red (parabolic), and green (elliptic)

Theorem 3 Let $W$ be an open set of $\mathbb{R}^{2}$ containing the origin and let $S: W \rightarrow \mathbb{R}^{2}$ be a real analytic map for which $(0,0)$ is a non-isolated fixed point, and such that $D S(0,0)=\left(\begin{array}{ll}1 & 1 \\ 0 & 1\end{array}\right)$. Suppose the set of fixed points of $S$ in $W$ is a real analytic curve $\mathcal{F}$, and let $T$ be a normal form of $S$ relative to $(0,0)$ and $W$. Let $Q$ and $\ell$ be associated to $T$ and $W$ as in Eq. (9).

If $Q=0$, then there exists a closed neighborhood $V \subset W$ of $(0,0)$ such that $V \backslash \mathcal{F}$ consists of two hyperbolic sectors relative to $(0,0)$ and $V$. If $Q \neq 0$, there exist a closed neighborhood $V$ of $(0,0)$ and a smooth curve $\Phi$ in $V$ with endpoints in the boundary of $V$ and tangential to $\mathcal{F}$ at $(0,0)$, such that $V \backslash(\mathcal{F} \cup \Phi)$ consists of four sectors relative to $(0,0)$ and $V$. Furthermore:

(a) If $\ell$ is odd, the four sectors, in either clockwise or counterclockwise orientation, are of elliptic, attracting parabolic, hyperbolic, and repelling parabolic type, respectively.

Also, $\Phi \backslash\{(0,0)\}$ has two connected components $\Phi^{u}$ and $\Phi^{s}$ such that $S^{-n}(x, y) \rightarrow(0,0)$ for every $(x, y) \in \Phi^{u}$ and $S^{n}(x, y) \rightarrow(0,0)$ for every $(x, y) \in \Phi^{s}$.

(b) If $Q(0)>0$ and $\ell$ is even, all four sectors are of repelling parabolic type, and $S^{-n}(x, y) \rightarrow(0,0)$ for every $(x, y) \in \Phi$.

(c) If $Q(0)<0$ and $\ell$ is even, all four sectors are of attracting parabolic type, and $S^{n}(x, y) \rightarrow(0,0)$ for every $(x, y) \in \Phi$.

The four cases in Theorem 3 are illustrated in Fig. 4. The proof of Theorem 3 will be given in Sect. 5.4. Before discussing the proof, we present some examples.

\subsection{Examples}

Example 1 (Continued) The map, for $a$ such that $0<a<1$, is

$$
F(x, y)=\left(\frac{x}{a+y}, \frac{y}{1+x}\right), \quad(x, y) \in[0, \infty) \times[0, \infty)
$$

In this example, the positive $y$ semi-axis is a curve of fixed points, and

$$
D F(0,1-a)=\left(\begin{array}{cc}
1 & 0 \\
(a-1) & 1
\end{array}\right)=J^{-1}\left(\begin{array}{ll}
1 & 1 \\
0 & 1
\end{array}\right) J, \quad \text { where } J=\left(\begin{array}{cc}
0 & \frac{1}{a-1} \\
1 & 0
\end{array}\right),
$$

so $(0,1-a)$ is a $1-1$ resonant fixed point of $F$. Translating $(0,1-a)$ to the origin and conjugating by $J$ yields the map in the form needed for Theorem 2 ,

$$
S(x, y)=\left(x+y-\frac{x y+y^{2}}{(a-1)+y}, y-\frac{x y}{1+x}\right) .
$$


We have $g(x, y)=-\frac{x y}{1+x}$ and $\varphi(x)=0$, so that by Theorem 3 ,

$$
Q(x) x^{\ell}=D_{y} g(x, 0)=\frac{-x}{1+x}=-x+x^{2}-x^{3}+\cdots .
$$

From Eq. (29), we see that $Q(0)=-1$ and $\ell=1$. By Theorem 3, the dynamics of Eq. (27) are conjugate to the dynamics pictured in Fig. 4(i), which agrees with the phase portrait in Fig. 1.

Example 2 (Continued) Consider the following map:

$$
F(x, y)=\left(\frac{x}{1+y}, \frac{x y}{x+y}\right), \quad x, y \in[0, \infty), x+y \neq 0
$$

In Eq. (30), the positive $x$-axis is a curve of fixed points, and

$$
D F(a, 0)=\left(\begin{array}{cc}
1 & -a \\
0 & 1
\end{array}\right)=J^{-1}\left(\begin{array}{ll}
1 & 1 \\
0 & 1
\end{array}\right) J, \quad \text { where } J=\left(\begin{array}{cc}
1 & 0 \\
0 & -\frac{1}{a}
\end{array}\right)
$$

so $(a, 0)$ is a $1-1$ resonant fixed point of $F$ for all $a>0$. Performing the change of coordinates $x \mapsto x+a$ and conjugating by $J$ gives the map

$$
S(x, y)=\left(\frac{a(x+y)}{a-y}, \frac{a(x+a) y}{(x+a)-y}\right) .
$$

We have $g(x, y)=\frac{a(x+a) y}{(x+a)-y}-y=\frac{y^{2}}{a(x+a)-y}$ and $\varphi(x)=0$, so that by Theorem 2,

$$
Q(x) x^{\ell}=D_{y} g(x, 0)=\left.\frac{y(2 a(x+a)-y)}{(a(x+a)-y)^{2}}\right|_{y=0}=0 .
$$

Thus $Q=0$, so by Theorem 3 the dynamical behavior of $(30)$ near $(a, 0)$ corresponds to (iv) of Fig. 4.

Example 3 Consider the planar map

$$
S(x, y)=\left(\begin{array}{c}
x+y-x^{2}+x^{3} \\
y-2 x^{3}+5 x^{4}-7 x^{5}+7 x^{6}-3 x^{7}+\left(2 x-4 x^{2}+8 x^{3}-6 x^{4}\right) y+(1-3 x) y^{2}
\end{array}\right) .
$$

For the map $S$ in (33), the set of fixed points is the graph of $\varphi(x)=x^{2}-x^{3}$. The point $(0,0)$ is a $1-1$ resonant fixed point of $S$ since $D S(0,0)=\left(\begin{array}{ll}1 & 1 \\ 0 & 1\end{array}\right)$. By Theorem 2 , we have

$$
\begin{aligned}
Q(x) x^{\ell} & =\left.D_{y}(g(x, y)-\varphi(x+y+f(x, y)))\right|_{y=\varphi(x)} \\
& =D_{y} g(x, \varphi(x))-\varphi^{\prime}(x+\varphi(x)+f(x, \varphi(x))) \cdot\left(1+D_{y} f(x, \varphi(x))\right) \\
& =4 x-x^{2}-\left(4 x-2 x^{2}-16 x^{3}+6 x^{4}+12 x^{5}-6 x^{6}\right) \\
& =x^{2}+16 x^{3}-6 x^{4}-12 x^{5}+6 x^{6} .
\end{aligned}
$$


Thus $Q(0)>0$ and $\ell=2$, so by Theorem 3 the local dynamical behavior of (33) is conjugate to the dynamics pictured in Fig. 4(ii).

\section{Proof of the main result}

\subsection{Overview}

By Theorem 1 and Theorem 2, a map $S$ with a $1-1$ resonant non-isolated fixed point $(\bar{x}, \bar{y})$ has a normal form and a real analytic curve of fixed points near $(\bar{x}, \bar{y})$. Thus, to prove Theorem 3, it suffices to do so for maps that are in normal form.

From now on, $T$ denotes a map in normal form defined in a neighborhood of the origin. In the proof we proceed by first establishing local dynamical behavior in the right-half plane, and then in the left-half plane. Given a map $S$, a set $A$ is $S$-invariant if $S(A) \subset A$.

The case when $Q(0)>0$ is considered first. Proposition 1 states that there is a $T^{-1}$ invariant curve in the first quadrant, one of whose endpoints is the origin. This curve is $C^{1}$ and tangential to $x$-axis at the origin. The curve separates first quadrant regions where $T$ has different dynamical behavior. In addition, points in the fourth quadrant belong to unstable invariant curves of fixed points on the axis. The case $Q(0)<0$ and general behavior on the left-half plane when $Q(0) \neq 0$ can be obtained from the study of the relationship between normal forms of a map and its inverse, which is the content of Lemma 1.

Proposition 1 uses Lemmas 2 and 3 to give dynamical behavior in the left-half plane. All the pieces are put together in Sect. 5.4, where the proof of Theorem 3 is completed, including details of the case when $Q=0$.

The proofs of Propositions 1 and 2 are given in Sects. 5.2 and 5.3, respectively.

Proposition 1 Let $T$ be a map in normal form (9). If $Q(0)>0$, then there exist $\delta>0$ and a $C^{1}$ curve $\phi^{u} \subset \mathcal{B}_{\delta} \cap \mathcal{Q}_{1}$ with one endpoint at $(0,0)$ and the other one on the line $x=\delta$ such that

(i) $\phi^{u}$ is $T^{-1}$-invariant.

(ii) $\phi^{u}=\left\{(x, y) \in \mathcal{B}_{\delta} \cap \mathcal{Q}_{1}: T^{-n}(x, y) \in \mathcal{B}_{\delta} \cap \mathcal{Q}_{1} \forall n \geq 0\right.$, and $\left.\lim _{n \rightarrow \infty} T^{-n}(x, y)=(0,0)\right\}$.

(iii) The $\operatorname{set}\left(\mathcal{B}_{\delta} \cap \mathcal{Q}_{1}\right) \backslash \phi^{u}$ has two connected components, henceforth denoted by $\mathcal{S}_{1}$ and $\mathcal{S}_{2}$, such that where $\mathcal{S}_{1}$ is a repelling parabolic sector of $T$ relative to $(0,0)$ and $\mathcal{B}_{\delta}$, and for $(x, y)$ in $\mathcal{S}_{2}$, both $T^{n}(x, y)$ and $T^{-n}(x, y)$ eventually leave $B_{\delta} \cap \mathcal{Q}_{1}$.

(iv) Every nonzero point $(x, y)$ in $\mathcal{B}_{\delta} \cap \mathcal{Q}_{4}$ belongs to the unstable invariant curve of a fixed point of $T$.

Proposition 2 Let $T$ be a map in normal form (9). If $Q(0)>0$ and $\ell$ is odd, or if $Q(0)<0$ and $\ell$ is even, then there exists $\delta>0$, a set $\mathcal{B}$ such that $\left(\mathcal{B}_{\delta} \cap \mathcal{Q}_{2}\right) \subset \mathcal{B} \subset \mathcal{Q}_{2}$, and a $C^{1}$ curve $\phi^{s} \subset \mathcal{B}$ with one endpoint at $(0,0)$ and the other one on the line $x=\delta$ such that

(i) $\phi^{s}$ is T-invariant.

(ii) $\phi^{s}=\left\{(x, y) \in \mathcal{B}_{\delta} \cap \mathcal{Q}_{2}: T^{n}(x, y) \in \mathcal{B}_{\delta} \cap \mathcal{Q}_{2} \forall n \geq 0\right.$, and $\left.\lim _{n \rightarrow \infty} T^{n}(x, y)=(0,0)\right\}$.

(iii) The set $\left(\mathcal{B}_{\delta} \cap \mathcal{Q}_{2}\right) \backslash \phi^{s}$ has two connected components, henceforth denoted by $\mathcal{S}_{1}^{\prime}$ and $\mathcal{S}_{2}^{\prime}$, where $\mathcal{S}_{1}^{\prime}$ is an attracting parabolic sector of $T$ relative to $(0,0)$ and $\mathcal{B}_{\delta}$, and for $(x, y)$ in $\mathcal{S}_{2}^{\prime}$, both $T^{n}(x, y)$ and $T^{-n}(x, y)$ eventually leave $\mathcal{B}_{\delta} \cap \mathcal{Q}_{2}$.

(iv) Every nonzero point $(x, y)$ in $\mathcal{B}_{\delta} \cap \mathcal{Q}_{3}$ belongs to the stable invariant curve of a fixed point of $T$ on the negative $x$ semi-axis.

Figure 5 depicts the behavior of solutions in the left- and right-hand planes described by Propositions 1 and 2 . 

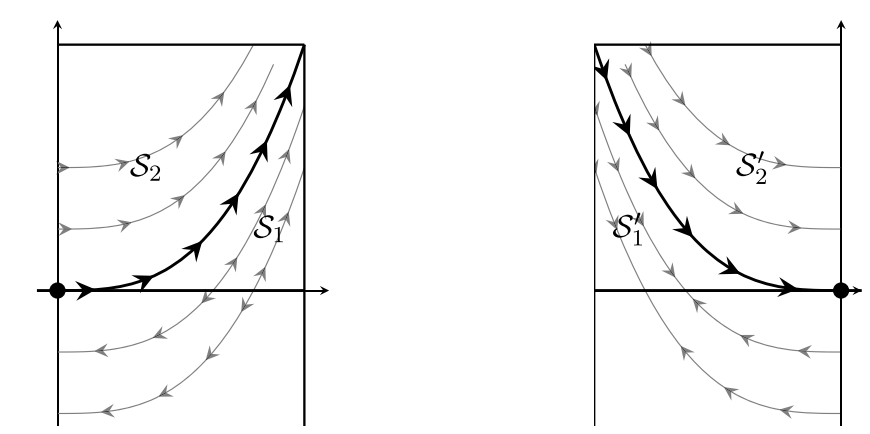

Figure 5 The regions $\mathcal{S}_{1}, \mathcal{S}_{2}, \mathcal{S}_{1}^{\prime}$, and $\mathcal{S}_{2}^{\prime}$ guaranteed by Propositions 1 and 2

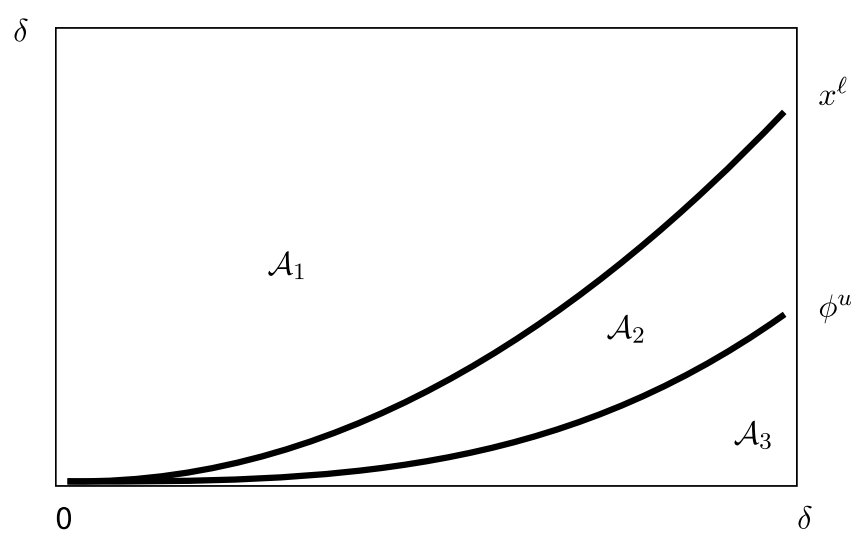

Figure 6 The region $[0, \delta] \times[0, \delta]$ is partitioned into three regions $\mathcal{A}_{1}, \mathcal{A}_{2}$, and $\mathcal{A}_{3}$

\subsection{Proof of Proposition 1}

If a map $T$ is in normal form (9), it is easy to see that $T(x, 0)=0, D T(0,0)=\left(\begin{array}{ll}1 & 1 \\ 0 & 1\end{array}\right)$, that $D^{j} T(0,0)=0$ for $1<j<\ell+1$ and $D_{x}^{\ell} D_{y} T_{2}(0,0)=\ell ! Q(0) \neq 0$. Thus Theorem A implies the existence (since $Q(0)>0 \Rightarrow b>0$ ) of an unstable invariant curve $\phi^{u}:[0, \delta) \rightarrow[0, \infty)$ where $\phi^{u}(x) \sim \frac{Q(0)}{\ell+1} x^{\ell+1}$ that is real analytic on the interior of its domain, establishing (i) and (ii) of Proposition 1 . Note that $\phi^{u}(x)<x^{\ell}$ for all $x \in[0, \delta]$ when $\delta$ is chosen sufficiently small.

It remains to establish the behavior of solutions lying in the region $\mathcal{B}_{\delta} \cap \mathcal{Q}_{1}$ off of $\phi^{u}$. To facilitate the proof, we break this region into three pieces. For each fixed $\delta>0$, define

$$
\begin{aligned}
& \mathcal{A}_{1}:=\left\{(x, y): 0 \leq x \leq \delta, x^{\ell}<y<\delta\right\}, \\
& \mathcal{A}_{2}:=\left\{(x, y): 0 \leq x \leq \delta, \phi^{u}(x)<y \leq x^{\ell}\right\}, \\
& \mathcal{A}_{3}:=\left\{(x, y): 0 \leq x \leq \delta, 0 \leq y \leq \phi^{u}(x)\right\},
\end{aligned}
$$

see Figure 6. It is clear that $\mathcal{A}_{1}, \mathcal{A}_{2}, \mathcal{A}_{3}$ depend upon $\delta>0$, but to ease the notation we will suppress this dependence. Lemma 2 below will show that $\mathcal{A}_{3}$ is a repelling hyperbolic sector with respect to the origin and $\mathcal{B}_{\delta}$ and Lemma 3 will show that $\mathcal{A}_{1} \cup \mathcal{A}_{2}$ is a subset of a hyperbolic sector with respect to the origin and $\mathcal{B}_{\delta}$. 
Lemma 2 If $\left(x_{0}, y_{0}\right) \in \mathcal{A}_{3}$, then $T^{-n}\left(x_{0}, y_{0}\right) \rightarrow(\bar{x}, 0)$ for some $\bar{x} \in(0, \delta)$.

Proof We begin by showing that the preimage of a vertical line segment lying in $\mathcal{A}_{3}$ is of the form $\mathcal{L}_{x_{0}}:=\left\{(x, y) \in \mathcal{A}_{3}: y=-x+x_{0}\right\}$. Suppose that $\mathcal{L}_{x_{0}}$ intersects $\phi^{u}$ at $\left(u, \phi^{u}(u)\right)$, so that the endpoints of $\mathcal{L}_{x_{0}}$ are $\left(u, \phi^{u}(u)\right)$ and $\left(x_{0}, 0\right)$. If $(x, y) \in \mathcal{L}_{x_{0}}$, then

$$
T_{1}(x, y)=x+y=x+\left(-x+x_{0}\right)=x_{0} .
$$

For points $(x, y) \in \mathcal{L}_{x_{0}}$, if $\delta>0$ is chosen sufficiently small, since both $x, x_{0}<\delta$,

$$
\begin{aligned}
\frac{\partial}{d \partial} T_{2}(x, y) & =\frac{\partial}{\partial x}\left(-x+x_{0}+Q(x) x^{\ell}\left(-x+x_{0}\right)+R\left(x,-x+x_{0}\right)\left(-x+x_{0}\right)^{2}\right) \\
& =-1+O(\delta)<0 .
\end{aligned}
$$

Equation (39) implies that $T_{2}(x, y)$ is monotone decreasing as $x$ increases along $\mathcal{L}_{x_{0}}$. By the invariance of $\phi^{u}$ and the $x$-axis under $T$, we see that $T\left(u, \phi^{u}(u)\right) \in \phi^{u}$ and $T(x, 0)=(x, 0)$, respectively. Thus

$$
0 \leq T_{2}(x, y) \leq T_{2}\left(u, \phi^{u}(u)\right), \quad \text { for all }(x, y) \in \mathcal{L}_{x_{0}} .
$$

By the continuity of $T_{2}$ and the Intermediate Value Theorem, for all $\left(x_{0}, y_{0}\right) \in \mathcal{A}_{3}$ there exists $(x, y) \in \mathcal{L}$ such that $T_{2}(x, y)=y_{0}$. By (38) we see that $T(x, y)=\left(x_{0}, y_{0}\right)$.

Now that we have established the preimage of the set $\left\{(u, v) \in \mathcal{A}_{3}: x=x_{0}\right\}$, we can use its structure to show that the $x$-coordinates of a backwards orbit of a point $\left(x_{0}, y_{0}\right) \in \mathcal{A}_{3}$ must converge. Let $\left(x_{n+1}, y_{n+1}\right):=T\left(x_{n}, y_{n}\right)$ for all $n \in \mathbb{Z}$. The Inverse Function Theorem tells us that $\left(x_{n}, y_{n}\right) \in \mathcal{B}_{\delta}$ has a unique preimage under $T$ in $\mathcal{B}_{\delta}$, thus

$$
\left(x_{n-1}, y_{n-1}\right)=T^{-1}\left(x_{n}, y_{n}\right) \in \mathcal{L}_{x_{n}} .
$$

Equation (41) shows that $\left\{x_{-n}\right\}$ is a decreasing sequence which is bounded below by 0 , so $\left\{x_{-n}\right\}$ converges to $\bar{x}$ for some $\bar{x}>0$. Turning our attention to the $y$-coordinate, for all $\left(x_{n}, y_{n}\right) \in \mathcal{A}_{3}$,

$$
y_{n+1}=y_{n}+Q\left(x_{n}\right) x_{n}^{\ell} y_{n}+R\left(x_{n}, y_{n}\right) y_{n}^{2}=y_{n}+\left(Q(0)+O(1) x_{n}\right) x_{n}^{\ell}>y_{n},
$$

so $\left\{y_{-n}\right\}$ is also a decreasing sequence bounded below by 0 , and thus converges to $\bar{y}$. The only fixed points in the closure of $\mathcal{A}_{3}$ lie on the $x$-axis, so $T^{-n}\left(x_{0}, y_{0}\right) \rightarrow(\bar{x}, 0)$.

Lemma 3 For every $\left(x_{0}, y_{0}\right) \in \mathcal{A}_{1} \cup \mathcal{A}_{2}$, there exists indices $m, k$ such that $T^{n}\left(x_{0}, y_{0}\right) \in$ $\mathcal{A}_{1} \cup \mathcal{A}_{2}$ for $-m \leq n \leq k$, and $T^{n}(x, y) \notin \mathcal{A}_{1} \cup \mathcal{A}_{2}$ for $n=-m-1$ and $n=k+1$.

Proof Define $\left(x_{n}, y_{n}\right):=T^{n}\left(x_{0}, y_{0}\right)$ for all $n \in \mathbb{Z}$. Suppose that $\left(x_{n}, y_{n}\right) \in \mathcal{A}_{1} \cup \mathcal{A}_{2}$. First, we narrow down the locations where $\left(x_{n+1}, y_{n+1}\right)$ may lie. Notice that $x_{n+1}=x_{n}+y_{n}$ and $x_{n}, y_{n}>$ 0 , so $\left(x_{n+1}, y_{n+1}\right)$ must lie in either $\mathcal{Q}_{1}$ or $\mathcal{Q}_{4}$. By (41), the unique preimages of points in $\mathcal{A}_{3}$ that lie in $\mathcal{B}_{\delta}$ must also lie in $\mathcal{A}_{3}$, so $\left(x_{n+1}, y_{n+1}\right) \notin \mathcal{A}_{3}$. To see that $y_{n+1}>0$, choose $\delta$ sufficiently small so that

$$
T_{2}\left(x_{n}, y_{n}\right)=y_{n}\left(1+Q\left(x_{n}\right) x_{n}^{\ell}+R\left(x_{n}, y_{n}\right) y_{n}\right)>0 \text {. }
$$


Equation (42) shows that $\left(x_{n+1}, y_{n+1}\right) \notin \mathcal{Q}_{4}$, so $\left(x_{n+1}, y_{n+1}\right) \in \mathcal{Q}_{1} \backslash \mathcal{A}_{3}$. Finally, suppose that $\left(x_{n}, y_{n}\right) \in \mathcal{A}_{1} \cup \mathcal{A}_{2}$ for all $n \in \mathbb{N}$. Then the sequence $\left\{x_{n}\right\}$ is increasing, and since $\left(x_{n}, y_{n}\right) \in$ $\mathcal{A}_{1} \cup \mathcal{A}_{2}$ and $\delta>0$ can be chosen such that $\phi^{u}(x)$ is an increasing function, we have $x_{n+1}-$ $x_{n}=y_{n} \geq \phi^{u}\left(x_{n}\right) \geq \phi^{u}\left(x_{0}\right)>0$. Then $\left\{x_{n}\right\}$ is not Cauchy and thus does not converge, so there must exist an index $k$ such that $\left(x_{k}, y_{k}\right) \in \mathcal{A}_{1} \cup \mathcal{A}_{2}$ and $x_{k+1}>\delta$, that is that $\left(x_{k+1}, y_{k+1}\right) \notin$ $\mathcal{A}_{1} \cup \mathcal{A}_{2}$, concluding the first part of the proof.

We will now show that there exists an index $m$ such that $T^{-m}\left(x_{0}, y_{0}\right) \in \mathcal{Q}_{2}$. Suppose that there exists $(x, y) \in \mathcal{A}_{1} \cup \mathcal{A}_{2}$ whose backwards orbit remains in $\mathcal{A}_{1} \cup \mathcal{A}_{2}$. In order to remain in $\mathcal{A}_{1}$ (and thus $\mathcal{Q}_{1}$ ), the sequence $\left\{x_{-n}\right\}$ must converge to some $\bar{x}$ since $\left\{x_{-n}\right\}$ is decreasing. This can happen only if $\left\{y_{-n}\right\} \rightarrow 0$. The only fixed point in the closure of $\mathcal{A}_{1} \cup \mathcal{A}_{2}$ is the origin, so our problem can be reduced to showing that the backwards orbit of any point in $\mathcal{A}_{1} \cup \mathcal{A}_{2}$ does not converge to the origin.

First, suppose for the sake of contradiction that $\left\{\left(x_{-n}, y_{-n}\right)\right\} \subset \mathcal{A}_{2}$ for all $n \in \mathbb{N}$. If $(x, y) \in$ $\mathcal{A}_{2}$, then $y=O\left(x^{\ell}\right)$, so for $\delta>0$ sufficiently small we have

$$
D_{x} T_{2}(x, y)=y\left(Q^{\prime}(x) x^{\ell}+Q(x) \ell x^{\ell-1}+D_{x} R(x, y) y\right) \geq y\left(Q(0) \ell x^{\ell-1}-O(1) x^{\ell}\right)>0 .
$$

Then it is easy to verify that every entry of the Jacobian matrix of $T$ is positive, and $T$ is orientation preserving in $\mathcal{A}_{2}$. Choose $(u, v) \in \phi^{u}$ such that $x \leq u$ and $y \geq v$. Since $T$ is orientation preserving in $\mathcal{A}_{2}$, the sequences $\left\{\left(x_{n}, y_{n}\right)\right\}$ and $\left\{\left(u_{n}, v_{n}\right)\right\}:=\left\{T^{n}(u, v)\right\}$ satisfy

$$
x_{-n} \leq u_{-n} \quad \text { and } \quad y_{-n} \geq v_{-n} \quad \text { for all } n \in \mathbb{N} .
$$

Since $(u, v) \in \phi^{u},\left\{\left(u_{-n}, v_{-n}\right)\right\} \rightarrow(0,0)$, which implies with $\left(x_{-n}, y_{-n}\right) \in \mathcal{A}_{2}$ and (44) that

$$
\left(x_{-n}, y_{-n}\right) \rightarrow(0,0) \quad \text { as } n \rightarrow \infty .
$$

From $\left(x_{n+1}, y_{n+1}\right)=T\left(x_{n}, y_{n}\right)$ and $\left(u_{n+1}, v_{n+1}\right)=T\left(u_{n}, v_{n}\right)$ it follows that

$$
u_{n+1}-x_{n+1}=u_{n}-x_{n}+v_{n}-y_{n} \leq u_{n}-x_{n} .
$$

From (44) and (46), $0<x_{0}-u_{0} \leq x_{-n}-u_{-n}$ for all $n \in \mathbb{N}$, which contradicts (45). Thus we can assume without loss of generality that $\left(x_{0}, y_{0}\right) \in \mathcal{A}_{1}$.

If $(x, y) \in \mathcal{A}_{1}$, then since $y \geq x^{\ell}$, we can choose $\delta>0$ sufficiently small such that

$$
x\left(T_{2}(x, y)-y\right)-y^{2}=y\left(Q(x) x^{\ell+1}+(x R(x, y)-1) y\right) \leq y\left(-x^{\ell}+O(1) x^{\ell+1}\right)<0 .
$$

Equation (47) implies that

$$
\frac{y_{n+1}}{x_{n+1}}=\frac{y_{n}+T_{2}\left(x_{n}, y_{n}\right)-y_{n}}{x_{n}+y_{n}}<\frac{y_{n}}{x_{n}} .
$$

It can easily be seen geometrically by considering the concavity of $y=x^{\ell}$ and relation (48) that $\left(x_{-n}, y_{-n}\right) \in \mathcal{A}_{1}$ for all $n \in \mathbb{N}$. Thus the sequence $\left\{y_{-n} / x_{-n}\right\}$ is increasing. Since $\left\{y_{-n} / x_{-n}\right\}$ 
is monotone, either $\left\{y_{-n} / x_{-n}\right\}$ converges to a constant strictly greater than 0 or $\left\{y_{-n} / x_{-n}\right\} \rightarrow$ $\infty$. Further we have

$$
\frac{y_{-n}}{x_{-n}}=\frac{\frac{y_{-n}}{x_{-n}}+\frac{y_{-n}}{x_{-n}}\left(Q\left(x_{-n}\right) x_{-n}^{\ell}+R\left(x_{-n}, y_{-n}\right) y_{-n}\right)}{1+\frac{y_{-n}}{x_{-n}}} .
$$

Set $\mu:=\lim y_{-n} / x_{-n}$ on the extended real line. Assuming that $\left(x_{-n}, y_{-n}\right) \rightarrow(0,0)$ and taking the limit as $n \rightarrow \infty$ to both sides, we have $\mu=\frac{\mu}{1+\mu}$, to which only $\mu=0$ is a solution on the extended real line, leading to a contradiction. Setting $\mathcal{S}_{1}:=\mathcal{A}_{3} \cap \mathcal{Q}_{\delta}$ and $\mathcal{S}_{2}:=\left(\mathcal{A}_{1} \cup \mathcal{A}_{2}\right) \cap$ $\mathcal{Q}_{\delta}$ completes the proof of part (iii) of Proposition 1.

Next, we will establish the dynamical behavior of solutions lying in $\mathcal{B}_{\delta} \cap \mathcal{Q}_{4}$. To begin, for each $\delta>0$ define

$$
\nabla_{\delta}:=\left\{(x, y) \in \mathcal{Q}_{4}: x-y<\delta\right\}
$$

We will construct a region satisfying part (iv) of Proposition 1 that lies inside $\nabla_{\delta}$. We wish to first show that orbits lying in $\nabla_{\delta}$ will eventually enter $\mathcal{Q}_{3}$. Choose $\delta>0$ so that, for all $(x, y) \in \nabla_{\delta}$,

$$
\begin{aligned}
& \frac{\left|T_{2}(x, y)-y\right|}{|y|}=\left|Q(x) x^{\ell}+R(x, y) y\right|<1, \\
& Q(x) x^{\ell}>0, \quad \text { and } \quad|x R(x, y)|<1,
\end{aligned}
$$

and so that the angle between the two eigenvectors of the jacobian of each of the fixed points $(x, 0) \in \nabla_{\delta}$ is less than $\frac{\pi}{4}$. This is possible because the angle $\theta(x)$ between the eigenvectors of the jacobian of $T$ at $(x, 0)$ are given by $\theta(x):=\arccos \left(1 / \sqrt{D_{y}\left(T_{2}(x, 0)\right)}\right)$, which satisfies $\theta \rightarrow 0$ as $x \rightarrow 0$. Notice that if $(x, y) \in \nabla_{\delta}$, by (51) and (52), respectively, we have

$$
T_{2}(x, y)=y\left(1+\frac{T_{2}(x, y)-y}{y}\right)<0
$$

and

$$
0>y\left(Q(x) x^{\ell+1}+(x R(x, y)-1) y\right)=x\left(T_{2}(x, y)-y\right)-y^{2} .
$$

Next, we must find the regions in which the image of a point $(x, y) \in \nabla_{\delta}$ may lie. By (53), $T(x, y)$ cannot lie in $\mathcal{Q}_{1} \cup \mathcal{Q}_{2}$. If $T(x, y) \in \mathcal{Q}_{4} \backslash \nabla_{\delta}$, then since $(x, y) \in \nabla_{\delta}$, we have $x<\delta+y$, which implies that

$$
\begin{aligned}
\delta & <(x+y)-T_{2}(x, y) \\
& =x-\left(Q(x) x^{\ell} y+R(x, y) y^{2}\right) \\
& <\delta+y+\left|Q(x) x^{\ell} y+R(x, y) y^{2}\right| \\
& =\delta-|y|+\left|Q(x) x^{\ell} y+R(x, y) y^{2}\right| .
\end{aligned}
$$


Equation (55) implies that

$$
|y|<\left|Q(x) x^{\ell} y+R(x, y) y^{2}\right|
$$

However, relation (51) implies that $|y|>\left|Q(x) x^{\ell} y+R(x, y) y^{2}\right|$, contradicting inequality (56). Thus $T(x, y) \in \nabla_{\delta} \cup \mathcal{Q}_{3}$. Now, we must rule out the possibility that an orbit remains in $\nabla_{\delta}$. Since $T_{1}(x, y)>0$ and $(x, y) \in \nabla_{\delta}, x\left(T_{2}(x, y)-y\right)-y^{2}<0 \Longleftrightarrow \frac{T_{2}(x, y)}{T_{1}(x, y)}<\frac{y}{x}$, so the angle formed between the $x$-axis and $T(x, y)$ is less than the angle formed between the $x$-axis and $(x, y)$. By an argument nearly identical to the one used to prove part (iii) of Proposition 1 , there exists an index $k \in \mathbb{N}$ such that $y_{k-1} / x_{k-1}<-1$, in which case $\left(x_{k}, y_{k}\right) \in \mathcal{Q}_{3}$.

By Theorem 5.1 in [20], the fixed point $(\delta, 0)$ has a local unstable invariant curve; define $\mathcal{U}_{\delta}$ to be the subset of the invariant curve that lies below the $x$-axis. The invariant curve $\mathcal{U}_{\delta}$ must be tangent to the eigenvector of the jacobian of $T$ at $(\delta, 0)$ which has vector angle less than $\pi / 4$. By the preceding paragraph, $T\left(\mathcal{U}_{\delta}\right) \subset \nabla_{\delta} \cup \mathcal{Q}_{3}$ and $T^{n}\left(\mathcal{U}_{\delta}\right) \cap \mathcal{Q}_{3} \neq \emptyset$ for $n$ sufficiently large. Thus $\mathcal{U}_{\delta}$ can be extended until it intersects the negative $y$-axis. Define $\mathcal{R}$ to be the region bounded by the $x$-axis, the $y$-axis, and $\mathcal{U}_{\delta}$.

It can easily be verified by (51) that if $(x, y) \in \mathcal{R}$, then $T^{-1}(x, y) \in \mathcal{Q}_{4}$. Consider any path from the origin to $\left(x_{-n}, y_{-n}\right)$ that is a subset of $\mathcal{R}$. Suppose that $T^{-1}\left(x_{-n}, y_{-n}\right) \notin \mathcal{R}$. Since the preimage of the path under $T$ must lie in $\mathcal{Q}_{4}$, it must intersect $\mathcal{U}_{\delta}$ at some point. This leads to a contradiction, since $\mathcal{U}_{\delta}$ is invariant under $T$ and the preimage is unique.

Now we have $\left\{\left(x_{-n}, y_{-n}\right)\right\} \subset \mathcal{R}$ for all $n \in \mathbb{N}$, so $\left\{x_{-n}\right\}$ is an increasing sequence which is bounded above by $\delta$. Using arguments analogous to those used in $\mathcal{A}_{3}$ in the proof of part (iii) of Proposition 1 shows that $T^{-n}(x, y) \rightarrow(\bar{x}, 0)$ for some $0<\bar{x}<\delta$. Choosing $\delta^{\prime}>0$ smaller if necessary such that $\mathcal{B}_{\delta^{\prime}} \subset \mathcal{R}$ completes the proof of part (iv) of Proposition 1 .

To study the left-half plane, we will focus on the scenario when $Q(0)>0$ and $\ell$ odd or when $Q(0)<0$ and $\ell$ even. The other combinations of signs for $Q(0)$ and parity of $\ell$ can be reduced to these cases through reflections about the origin.

\subsection{Proof of Proposition 2}

Theorem A implies the existence of a stable invariant curve $\phi^{s}:(-\delta, 0] \rightarrow(-\infty, 0]$ by the same argument used at the beginning of the proof of Proposition 1. (Recall that $\ell=n-1$, so $\ell$ odd implies $n$ even and vice versa.) By Corollary 1 , it is possible to choose $\Theta$ and $\delta^{\prime}>0$ such that $\Theta\left(\mathcal{B}_{\delta^{\prime}} \cap \mathcal{Q}_{2}\right) \subset \mathcal{B}_{\delta} \cap \mathcal{Q}_{1}$. Set $\mathcal{S}_{1}^{\prime}=\Theta^{-1}\left(\mathcal{S}_{1}\right) \cap \mathcal{B}_{\delta^{\prime}}$ and $\mathcal{S}_{2}^{\prime}=\Theta^{-1}\left(\mathcal{S}_{2}\right) \cap \mathcal{B}_{\delta^{\prime}}$. Using a normal form of $T^{-1}$, it is not difficult to verify that these sets have the properties claimed by Proposition 2, so the proof is omitted.

\subsection{Proof of Theorem 3}

Let $T$ be a map in normal form, defined on a neighborhood $V$ of the origin. There are some properties of $T$ that follow immediately from (9). In particular, every point in $V$ of the form $(x, 0)$ is a fixed point of $T$, and any sufficiently small neighborhood $V$ of the origin has the property

$$
(x, y) \in V \Longrightarrow\left\{\begin{array}{llll}
x<T_{1}(x, y) & \text { and } & T_{2}(x, y)>0 & \text { whenever } y>0, \\
x>T_{1}(x, y) & \text { and } & T_{2}(x, y)<0 & \text { whenever } y<0 .
\end{array}\right.
$$


To establish additional dynamical behavior characteristics of $T$ near the origin takes considerably more work. Next, we will combine Propositions 1 and 2 to see the complete dynamical picture. There are four non-conjugate dynamic scenarios; we will consider each one separately.

Case 1: $Q(0)>0$ and $\ell$ odd. If $Q(0)>0$ and $\ell$ is odd, then the behavior in the right- and left-half planes are described by Propositions 1 and 2, respectively. Let $\phi=\phi^{s} \cup \phi^{u}$. Since $\phi^{s}$ and $\phi^{u}$ are both tangential to the $x$-axis at the origin, $\phi$ is a $C^{1}$ manifold. Then $\mathcal{S}_{1}$ and $\mathcal{S}_{1}^{\prime}$ are, respectively, repelling and attracting parabolic sectors with respect to the origin and $\mathcal{B}_{\delta}$.

We now show that the intersection of certain neighborhood of the origin with the lowerhalf plane is an elliptic sector. Choose $\delta>0$ so that, for all $(x, y) \in V$ satisfying $\|(x, y)\|_{1}<\delta$, we have $\left|Q(x) x^{\ell}+R(x, y) y\right|<1$. Then if $\|(x, y)\|_{1}<\delta$,

$$
\begin{aligned}
\|T(x, y)\|_{1} & =|x+y|+\left|T_{2}(x, y)\right| \\
& \leq|x|+|y|+|y|\left|1+Q(x) x^{\ell}+R(x, y) y\right|<3 \delta .
\end{aligned}
$$

By Proposition 1, if $(x, y) \in \mathcal{B}_{\delta} \cap \mathcal{Q}_{4}$ and $\|(x, y)\|_{1}<\delta$, then there exists an index $N \in \mathbb{N}$ such that $T^{N}(x, y) \in \mathcal{Q}_{3}$, and $\left\|T^{n}(x, y)\right\|_{1}<\delta$ for all $1 \leq n \leq N-1$. By Proposition 2 , for $\delta>0$ sufficiently small, if $(x, y) \in \mathcal{B}_{\delta} \cap \mathcal{Q}_{3}$, then $(x, y)$ lies on a stable invariant curve of a fixed point on the negative $x$-axis. Choose $\delta>0$ such that $\mathcal{B}_{3 \delta} \subset V$. Then, for the open $\operatorname{disk} B((0,0), \delta)$, if $(x, y) \in B((0,0), \delta) \cap \mathcal{Q}_{4}$, then the orbit of $(x, y)$ converges to a fixed point on the negative $x$-axis. We already know by Proposition 1 that $(x, y)$ lies on an unstable invariant curve of a fixed point on the positive $x$-axis. A similar argument for $\mathcal{B}_{\delta} \cap \mathcal{Q}_{3}$ shows that $\mathcal{B}_{\delta} \cap \mathbb{Y}_{-}$is an elliptic sector relative to $(0,0)$.

Finally, we claim that if $\mathcal{S}_{2}$ and $\mathcal{S}_{2}^{\prime}$ are defined as in Propositions 1 and 2 , then $\mathcal{S}_{2} \cup \mathcal{S}_{2}^{\prime}$ is a hyperbolic sector. By Proposition 2, if $(x, y) \in \mathcal{S}_{2}$, then there exist indices $m, k \in \mathbb{N}$ such that $T^{k}(x, y) \notin \mathcal{B}_{\delta} \cap \mathcal{Q}_{1}$ and $T^{-m}(x, y) \notin \mathcal{B}_{\delta} \cap \mathcal{Q}_{1}$. By $(57), T^{k}(x, y) \notin \mathcal{Q}_{2}$. If $T^{-m}(x, y) \in \mathcal{B}_{\delta} \cap \mathcal{Q}_{2}$, then Proposition 1 implies that there exists an index $m^{\prime} \in \mathbb{N}$ such that $T^{-m^{\prime}}\left(T^{-m}(x, y)\right) \notin$ $\mathcal{B}_{\delta} \cap \mathcal{Q}_{2}$. By $(57), T^{-\left(m+m^{\prime}\right)}(x, y) \notin \mathcal{Q}_{1}$, and thus $\mathcal{S}_{1} \cup \mathcal{S}_{1}^{\prime}$ is a hyperbolic sector with respect to the origin and $\mathcal{B}_{\delta}$, completing Case 1 .

Case 2: $Q(0)<0$ and $\ell$ odd. If $Q(0)<0$ and $\ell$ is odd, then reflecting about the origin gives a system in which $Q(0)>0$ and $\ell$ is odd, so this situation is conjugate to the first case.

Case 3: $Q(0)>0$ and $\ell$ even. If $Q(0)>0$ and $\ell$ is even, then the behavior in the right-half plane is described by Proposition 1 . Further, reflecting about the origin gives a system in which $Q(0)>0$ and $\ell$ is even, so the dynamical behavior in the left-half plane is equivalent to the dynamics of the right-half plane after a reflection about the origin. Let $\hat{\phi}^{u}$ be the invariant curve (lying in $\mathcal{Q}_{3}$ after reflection about the origin) guaranteed by Proposition 1 and let $\phi:=\phi^{u} \cup \hat{\phi}^{u}$. Since the one-sided derivatives of both $\phi^{u}$ and $\hat{\phi}^{u}$ are zero at the origin, $\phi$ is a manifold. We see immediately that $\mathcal{S}_{1}$ and its reflection about the origin are two repelling parabolic sectors.

We wish to show the existence of two more repelling parabolic sectors. By Proposition 2, if $(x, y) \in \mathcal{B}_{\delta} \cap \mathcal{Q}_{2}$, then $(x, y)$ lies in the unstable invariant curve of a fixed point $(\bar{x}, 0)$ lying on the negative $x$ semi-axis. We claim that these unstable invariant curves can be extended to intersect the set $\mathcal{B}_{\delta} \cap \mathcal{Q}_{1}$. Consider an unstable invariant curve through the point $\left(0, \delta^{\prime}\right)$ for $\delta^{\prime}>0$, which is guaranteed by Proposition 1 . Since $T_{1}\left(0, \delta^{\prime}\right)=\delta^{\prime}$, the unstable invariant 
Figure 7 The region $\mathcal{D}$. The upper curve is the unstable manifold that contains the point $\left(0, \delta^{\prime}\right)$

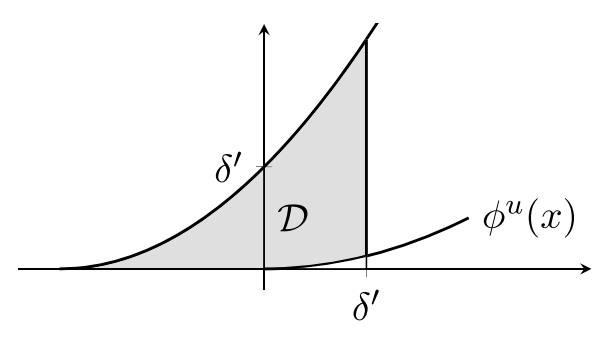

curve through $\left(0, \delta^{\prime}\right)$ intersects any vertical line $x=x_{0}$ with $0<x_{0} \leq \delta^{\prime}$. Let $\mathcal{D}$ be the open region bounded by the unstable invariant curve, the vertical line $x=\delta^{\prime}, \phi^{u}$, and the $x$-axis, see Figure 7. By the Inverse Function Theorem, we can ensure with an appropriately small choice of $\delta^{\prime}>0$ that $T$ is injective in $\mathcal{D}$, and that $\mathcal{D} \subset V$. We claim that, for any $(x, y) \in \mathcal{D}$, $T^{-n}(x, y) \in \mathcal{D}$. To prove the claim, consider any curve connecting the origin to $(x, y)$ for which all points on the curve other than the endpoints are contained completely within the interior of $\mathcal{D}$. Then, by (57), the preimages of any points on the curve under $T$ have $x$-coordinates less than $\delta^{\prime}$, so if $T^{-n}(x, y) \notin \mathcal{D}$, there exists a point lying on the curve whose preimage under $T$ lies on either $\phi^{u}$, the invariant curve, or the $x$-axis. In any case, this contradicts the invariance of these sets under $T^{-1}$. Thus $\left\{T^{-n}(x, y)\right\}$ lies in $\mathcal{D}$ for all $n \geq 0$. By (57), $\left\{x_{n}\right\}$ is a monotone decreasing sequence that is bounded below, and thus converges. This implies that $\left\{T^{-n}(x, y)\right\}$ converges to a point lying in the negative $x$ semi-axis. Then choosing $\delta>0$ such that $\mathcal{S}_{2} \subset \mathcal{D}$ shows that $\left(\mathcal{B}_{\delta} \cap \mathcal{Q}_{2}\right) \cup \mathcal{S}_{2}$ is a repelling parabolic sector with respect to the origin and $\mathcal{B}_{\delta}$. A similar argument holds for the remaining sector.

Case 4: $Q(0)<0$ and $\ell$ odd. The proof is similar to the proof of the case $Q(0)>0$ and $\ell$ even, so we skip it.

Case 5: $Q=0$. We wish to show that, for any $\delta>0$ sufficiently small, if $(x, y) \in \mathcal{B}_{\delta} \cap \mathbb{Y}_{+}$ or $\mathcal{B}_{\delta} \cap \mathbb{Y}_{-}$, then both $\left\{T^{n}(x, y)\right\}$ and $\left\{T^{-n}(x, y)\right\}$ eventually leave $\mathcal{B}_{\delta} \cap \mathbb{Y}_{+}$or $\mathcal{B}_{\delta} \cap \mathbb{Y}_{-}$. Let $T^{n}(x, y):=\left(x_{n}, y_{n}\right)$. A contradiction is reached by showing that an orbit remaining in $\mathcal{B}_{\delta}$ must converge to a point on the $x$-axis, and then, by showing that no orbit can converge to the $x$-axis. Choose $\delta>0$ such that

$$
|R(x, y) y|<1 \quad \text { for all }(x, y) \in \mathcal{B}_{\delta} .
$$

Suppose that $\left(x_{n}, y_{n}\right) \in \mathcal{B}_{\delta}$ for all $n \geq 1$. Then, by the definition of $T$,

$$
\left|x_{n+1}-x_{n}\right|=\left|y_{n}\right| \quad \text { for all } n \geq 1
$$

By (57), $\left\{x_{n}\right\}$ is a monotone sequence that is bounded by $\pm \delta$, so $\left\{x_{n}\right\}$ converges, say to $\bar{x}$. Then (60) implies that $\left\{y_{n}\right\}$ converges to 0 . Thus $\left\{\left(x_{n}, y_{n}\right)\right\} \in \mathcal{B}_{\delta}$ for all $n \in \mathbb{N}$ implies that $\left\{\left(x_{n}, y_{n}\right)\right\}$ converges to a point $(\bar{x}, 0) \in \mathcal{B}_{\delta}$.

We now show that $\left\{\left(x_{n}, y_{n}\right)\right\}$ cannot converge to $(\bar{x}, 0)$ for $\left\{\left(x_{n}, y_{n}\right)\right\} \subset \mathcal{B}_{\delta} \cap \mathbb{Y}_{+}$and $\bar{x}>0$; the proof for the remaining combinations of $\bar{x}>0, \bar{x}<0, \mathbb{Y}_{+}$, and $\mathbb{Y}_{-}$are similar. If $\left\{x_{n}\right\}$ converges to $\bar{x}$, then, by (57), it follows that $x_{n}-\bar{x}<0$. This along with inequality (59) yields

$$
0>\left(x_{n}-\bar{x}\right) y_{n}\left(1+R\left(x_{n}, y_{n}\right) y_{n}\right)-y_{n}^{2},
$$


and since $\left(x_{n}, y_{n}\right) \rightarrow(\bar{x}, 0)$, then by (57) we have additionally $0>T_{1}\left(x_{n}, y_{n}\right)-\bar{x}$, so it can be verified that

$$
\frac{T_{2}\left(x_{n}, y_{n}\right)}{T_{1}\left(x_{n}, y_{n}\right)-\bar{x}}=\frac{y_{n}\left(1+R\left(x_{n}, y_{n}\right) y_{n}\right)}{x_{n}-\bar{x}+y_{n}}<\frac{y_{n}}{x_{n}-\bar{x}} .
$$

In the light of Eqs. (61) and (62), the slopes of the line segment between $\left(x_{n}, y_{n}\right)$ and $(\bar{x}, 0)$ form a decreasing sequence of negative terms, and thus converge to a fixed negative constant in the extended real line. An argument analogous to that used in the proof of (iii) of Proposition 1 shows that $\left\{\left(x_{n}, y_{n}\right)\right\}$ cannot converge to $(\bar{x}, 0)$. A similar argument can be used for the remaining cases of $\left\{\left(x_{n}, y_{n}\right)\right\}$ in the upper- and lower-half planes and $(\bar{x}, 0)$ in the positive or negative $x$ semi-axes. Thus $\left\{T^{n}(x, y)\right\}$ must eventually leave $\mathcal{B}_{\delta}$.

Applying Lemma 1 together with Corollary 1 and repeating the argument above with $\tilde{T}$ shows that $\left\{T^{-n}(x, y)\right\}$ must eventually leave $\mathcal{B}_{\delta}$, which shows that $\mathcal{B}_{\delta} \cap \mathbb{Y}_{+}$and $\mathcal{B}_{\delta} \cap \mathbb{Y}_{-}$are both hyperbolic sectors relative to $(0,0)$ and $V$.

\footnotetext{
Acknowledgements

The authors would like to thank the anonymous referee, whose comments improved this article.
}

Competing interests

The authors declare that they have no competing interests.

Authors' contributions

Both authors contributed equally to this work. They both read and approved the submitted manuscript.

\section{Author details}

'Department of Mathematics, S. New Hampshire University, Manchester, USA. ${ }^{2}$ Department of Mathematics, University of Rhode Island, Kingston, USA.

\section{Publisher's Note}

Springer Nature remains neutral with regard to jurisdictional claims in published maps and institutional affiliations.

Received: 11 December 2017 Accepted: 10 April 2018 Published online: 20 April 2018

\section{References}

1. Smith, H.: Invariant curves for mappings. SIAM J. Math. Anal. 17, 1053-1067 (1986)

2. Elaydi, S.: An Introduction to Difference Equations, 2nd edn. Springer, New York (1999)

3. Agarwal, R.P.: Difference Equations and Inequalities. Dekker, New York (1992)

4. Kulenović, M.R.S., Ladas, G.: Dynamics of Second Order Rational Difference Equations. Chapman and Hall/CRC, Boca Raton (2002)

5. Kulenović, M.R.S., Merino, O.: Discrete Dynamical Systems and Difference Equations with Mathematica. Chapman and Hall/CRC, Boca Raton (2002)

6. Kulenović, M.R.S., Nurkanović, Z., Pilav, E.: Birkhoff normal forms and KAM theory for Gumowski-Mira equation. Sci. World J. 2014, Article ID 819290 (2014)

7. Kuznetsov, Y.A.: Elements of Applied Bifurcation Theory, 2nd edn. Springer, New York (1998)

8. Casasayas, J., Fontich, E., Nunes, A.: Invariant manifolds for a class of parabolic points. Nonlinearity 5, 1193-1210 (1992)

9. Clark, D., Kulenović, M.R.S.: A coupled system of rational difference equations. Comput. Math. Appl. 43, 849-867 (2002)

10. Clark, D., Kulenović, M.R.S., Selgrade, J.: Global asymptotic behavior of a two-dimensional difference equation modelling competition. Nonlinear Anal. 52, 1765-1776 (2003)

11. Hassell, M.: The Spatial and Temporal Dynamics of Host-Parasitoid Interactions. Oxford University Press, London (2000)

12. Nicholson, A.J., Bailey, V.A.: The balance of animal populations. J. Zool. 109, 551-598 (1935). https://doi.org/10.1111/j.1096-3642.1935.tb01680.x

13. Rogers, D.J.: Random search and insect population models. J. Anim. Ecol. 41(2), 369-383 (1972). https://doi.org/10.2307/3474

14. May, R.M.: Host-parasitoid systems in patchy environments: a phenomenological model. J. Anim. Ecol. 47, 833-844 (1978)

15. McArdle, D., Merino, O.: Global dynamics of a Leslie host-parasite model. J. Differ. Equ. Appl. 24, 82-106 (2018). https://doi.org/10.1080/10236198.2017.1397139

16. Din, Q., Donchev, T.: Global character of a host-parasite model. Chaos Solitons Fractals 54, 1-7 (2013) 
17. Leslie, P.H.: Some further notes on the use of matrices in population mathematics. Biometrika 55, 213-245 (1948)

18. Krantz, S., Parks, H.: A Primer of Real Analytic Functions. Birkhäuser, Basel (1992)

19. Gubar, N.A.: Investigation by Bendixson's method of the topological structure of the configuration of paths in the neighborhood of a singular point of a certain dynamic system. Izv. Vysš. Učebn. Zaved., Radiofiz. 2, Article ID 6 (1959) (Russian)

20. Hirsch, M.W., Pugh, C.C., Shub, M.: Invariant Manifolds. Lecture Notes in Mathematics, vol. 583. Springer, New York (1977)

Submit your manuscript to a SpringerOpen ${ }^{\circ}$ journal and benefit from:

- Convenient online submission

- Rigorous peer review

- Open access: articles freely available online

- High visibility within the field

- Retaining the copyright to your article

Submit your next manuscript at $\boldsymbol{\Delta}$ springeropen.com 